



\section{Historic, archived document}

Do not assume content reflects current scientific knowledge, policies, or practices 


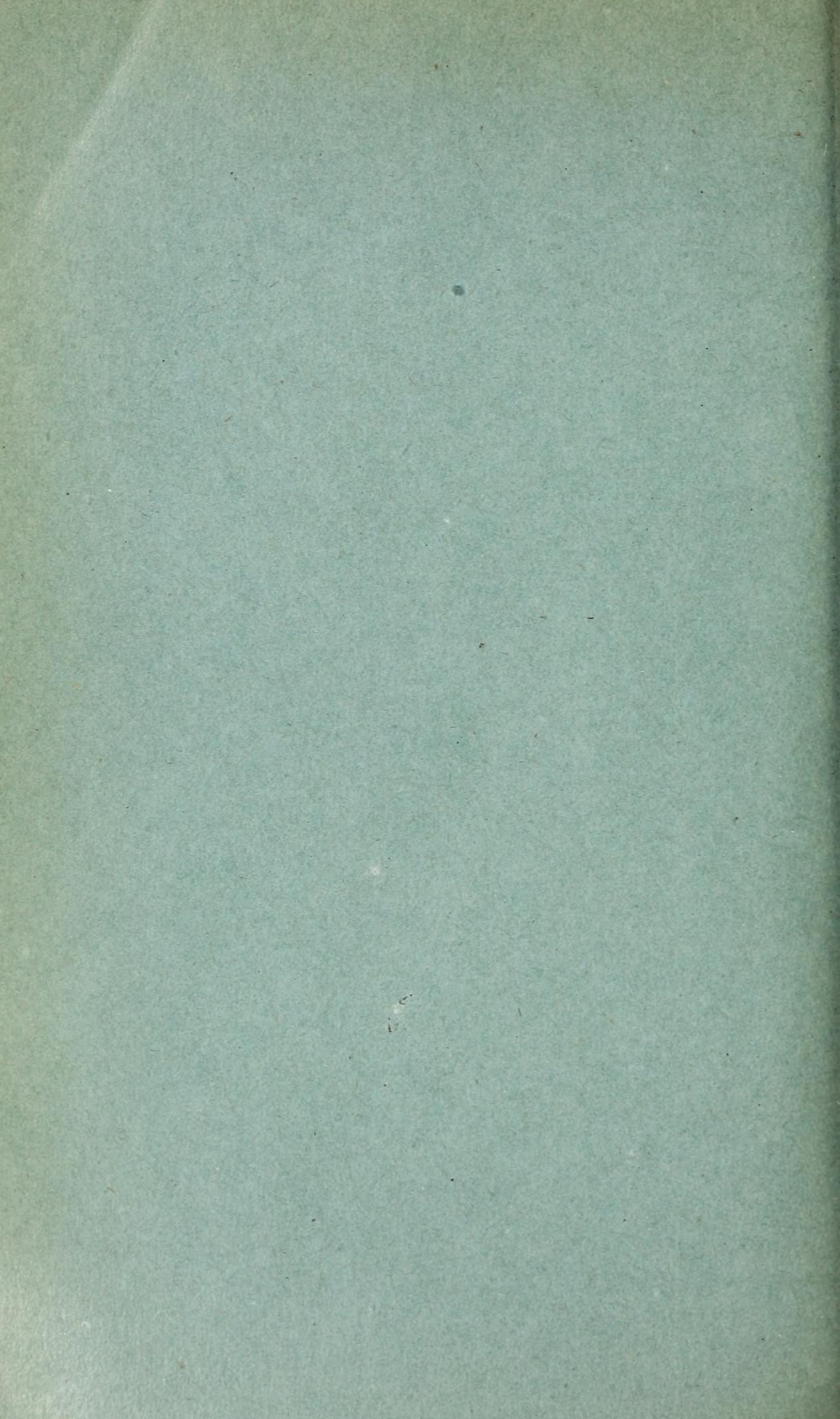




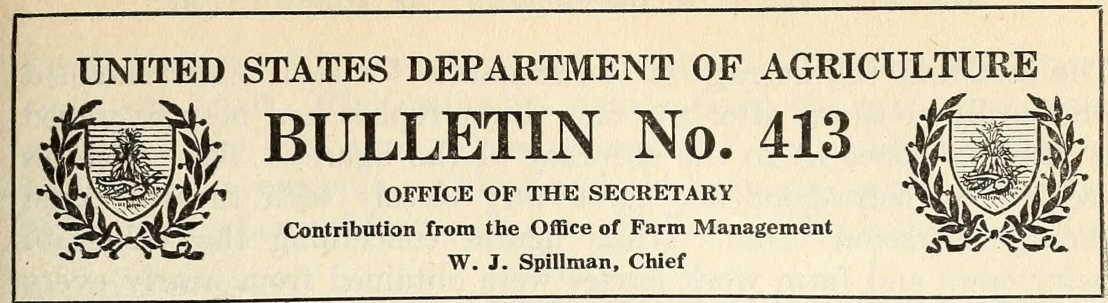

Washington, D. C.

PROFESSIONAL PAPER

August 24, 1916

\title{
INFLUENCE OF AGE ON THE VALUE OF DAIRY COWS AND FARM WORK HORSES.
}

\author{
By J. C. McDowell, \\ Agriculturist, Office of Farm Management.
}

CONTENTS.

Page.

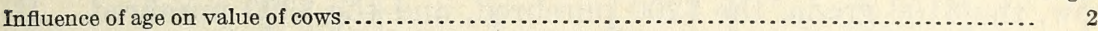

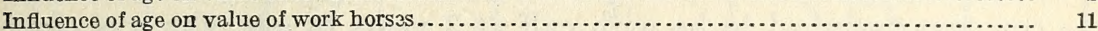

Research work in farm economics includes among other things questions relating to the value of farm animals, farm equipment, and agricultural products. Those who have occasion to buy or sell live stock are constantly confronted with the problem of weighing the many factors influencing the value of farm animals. Of these factors, the age of the animal is one that applies directly to the value of dairy cattle and farm work horses. While age is not the only factor affecting the value of these classes of animals, it is one of the most important. In determining the value of farm animals grown for meat only, which are ordinarily disposed of at an early age, this factor is comparatively unimportant.

By means of personal visits and through correspondence with many of our best informed farmers and live-stock breeders, the Office of Farm Management recently attempted to get reliable information concerning the influence of age on the values of dairy cows and farm work horses. Farm Management investigators have found that for farm data the average of a large number of estimates is almost as accurate as that of carefully kept records, provided the estimates are given by men who thoroughly know the subject under consideration. In this case all estimates were given by men each of whom has had wide experience with the class of live stock on which he furnishes information. 
The fact that the average of the estimates for each class remained practically constant after the first thirty replies had been averaged is some evidence as to the accuracy of the figures. The estimates secured by correspondence agree very closely with those secured through personal visits. While figures concerning the values of dairy cows and farm work horses were obtained from nearly every important agricultural district in the United States, most of the estimates were secured from New York, Pennsylvania, and the North Central States. In this work appreciation and depreciation were based on age only. In order to eliminate everything except age, the animal was assumed to be in perfect health and to have a fixed value at a certain age.

Four breeds of dairy cattle, Holstein, Guernsey, Jersey, and Ayrshire, were included in these investigations. No attempt was made to find the actual average value of animals belonging to any breed. Arbitrary values were assumed for a certain fixed age, merely to get a starting point from which to work. Thus for each breed estimates were based on four classes of 3-year-olds, namely, the $\$ 80$ grade cow, the $\$ 100$ grade, the $\$ 200$ purebred, and the $\$ 300$ purebred. If the cow was assumed to be with calf at 3 years of age, she was considered to have reached the same stage of pregnancy each succeeding year. In the case of purebred stock changes in the advanced registry of the cow herself, or of other animals related to her, were not considered in estimating values.

The farm work horse was assumed to have a maximum value of $\$ 250$ when in his prime, which is a fair price for very good farm horses. Brood mares were excluded, as their value is not necessarily the same as that of animals kept for work only.

In so far as age affects values the tables presented in the bulletin should be helpful in buying and selling dairy cows and farm work horses, and in taking inventories. No attempt has been made to compare breeds. In fact, it is believed that none of the figures can be interpreted in such a way as to favor any particular breed.

\section{INFLUENCE OF AGE ON VALUE OF COWS.}

\section{HOLSTEIN.}

Table I shows the influence of age on the value of four classes of Holstein cows worth per head $\$ 80, \$ 100, \$ 200$, and $\$ 300$, respectively, at 3 years of age. (See fig. 1.) For each class the estimates are tabulated separately for the North Central States, the Northeastern States, and the United States, all sections. According to these estimates Holstein cows of all classes reach their maximum money value at 6 years of age. The values do not vary 
greatly between the ages of 4 and 8 years. After that age depreciation is rapid. The estimated values given old cows may seem a little high, but it must be remembered that only animals in health were considered. Calves and old cows are valued relatively higher in the Central States than in the East. This is doubtless due

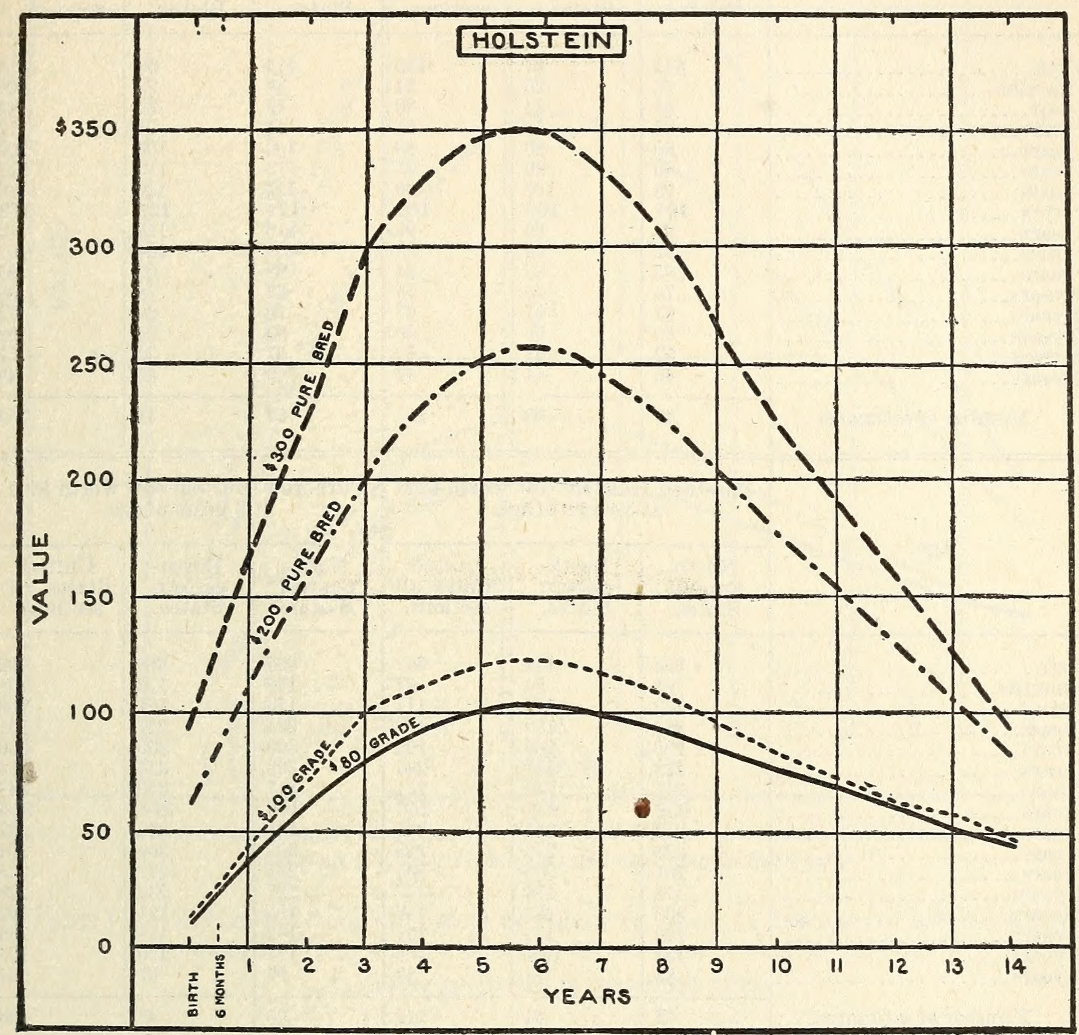

FIG. 1.-Curves showing influence of age on values of Holstein cows. (Averages of 528 estimates by owners, based on arbitrary values assumed for a certain fixed age.)

largely to the lower price of feed in the North Central States. During their years of highest production, the price of Holstein cows appears to average a little higher in the Eastern States. This difference, however, is not marked, and in the case of the $\$ 100$ grade cow it is reversed. 
TABLE I.-Influence of age on values of Holstein cows. (Averages of 528 estimates by owners, based on arbitrary values assumed for a certain fixed age.)

[Averages are reported to the nearest dollar.]

\begin{tabular}{|c|c|c|c|c|c|c|}
\hline \multirow{2}{*}{ Age. } & \multicolumn{3}{|c|}{$\begin{array}{l}\text { Grade Holstein cow worth } \$ 80 \\
\text { at } 3 \text { years of age. }\end{array}$} & \multicolumn{3}{|c|}{$\begin{array}{c}\text { Grade Holstein cow worth } \$ 100 \\
\text { at } 3 \text { years of age. }\end{array}$} \\
\hline & $\begin{array}{l}\text { North } \\
\text { Central } \\
\text { States. }\end{array}$ & $\begin{array}{l}\text { North- } \\
\text { eastern } \\
\text { States. }\end{array}$ & $\begin{array}{l}\text { United } \\
\text { States, all } \\
\text { sections. }\end{array}$ & $\begin{array}{l}\text { North } \\
\text { Central } \\
\text { States. }\end{array}$ & $\begin{array}{l}\text { North- } \\
\text { eastern } \\
\text { States. }\end{array}$ & $\begin{array}{l}\text { United } \\
\text { States, all } \\
\text { sections. }\end{array}$ \\
\hline \multirow[t]{2}{*}{ 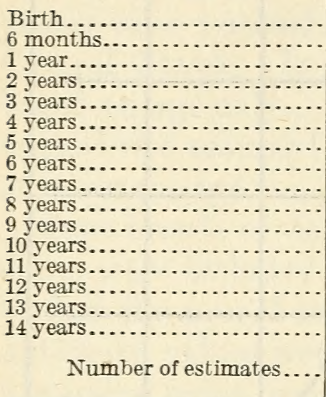 } & $\begin{array}{r}\$ 12 \\
28 \\
43 \\
63 \\
80 \\
90 \\
98 \\
\mathbf{1 0 1} \\
98 \\
92 \\
82 \\
74 \\
67 \\
60 \\
52 \\
46\end{array}$ & $\begin{array}{r}\$ 7 \\
20 \\
34 \\
58 \\
80 \\
90 \\
100 \\
\mathbf{1 0 2} \\
99 \\
93 \\
85 \\
76 \\
67 \\
59 \\
51 \\
44\end{array}$ & $\begin{array}{r}\$ 10 \\
24 \\
39 \\
61 \\
80 \\
91 \\
100 \\
\mathbf{1 0 2} \\
99 \\
93 \\
84 \\
75 \\
67 \\
60 \\
52 \\
44\end{array}$ & $\begin{array}{r}\$ 13 \\
30 \\
49 \\
75 \\
100 \\
113 \\
121 \\
121 \\
116 \\
109 \\
98 \\
87 \\
75 \\
65 \\
57 \\
49\end{array}$ & $\begin{array}{r}\$ 9 \\
22 \\
39 \\
71 \\
100 \\
110 \\
120 \\
122 \\
115 \\
104 \\
92 \\
78 \\
69 \\
60 \\
52 \\
46\end{array}$ & $\begin{array}{r}\$ 11 \\
26 \\
44 \\
72 \\
100 \\
112 \\
120 \\
122 \\
116 \\
107 \\
95 \\
83 \\
72 \\
62 \\
57 \\
48\end{array}$ \\
\hline & 49 & 48 & 107 & 57 & 41 & $\overline{159}$ \\
\hline \multirow{2}{*}{ Age. } & \multicolumn{3}{|c|}{$\begin{array}{l}\text { Purebred Holstein cow worth } \$ 200 \\
\text { at } 3 \text { years of age. }\end{array}$} & \multicolumn{3}{|c|}{$\begin{array}{l}\text { Purebred Holstein cow worth } \$ 300 \\
\text { at } 3 \text { years of age. }\end{array}$} \\
\hline & $\begin{array}{l}\text { North } \\
\text { Central } \\
\text { States. }\end{array}$ & $\begin{array}{l}\text { North- } \\
\text { eastern } \\
\text { States. }\end{array}$ & $\begin{array}{l}\text { United } \\
\text { States, all } \\
\text { sections. }\end{array}$ & $\begin{array}{l}\text { North } \\
\text { Central } \\
\text { States. }\end{array}$ & $\begin{array}{l}\text { North- } \\
\text { eastern } \\
\text { States. }\end{array}$ & $\begin{array}{l}\text { United } \\
\text { States, all } \\
\text { sections. }\end{array}$ \\
\hline 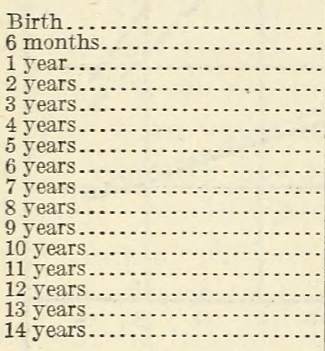 & $\begin{array}{r}\$ 64 \\
91 \\
122 \\
150 \\
200 \\
228 \\
245 \\
252 \\
242 \\
226 \\
201 \\
174 \\
152 \\
128 \\
104 \\
84\end{array}$ & $\begin{array}{r}\$ 59 \\
84 \\
113 \\
159 \\
200 \\
231 \\
249 \\
\mathbf{2 5 7} \\
245 \\
229 \\
203 \\
179 \\
153 \\
126 \\
101 \\
73\end{array}$ & $\begin{array}{r}\$ 62 \\
87 \\
117 \\
160 \\
200 \\
233 \\
251 \\
256 \\
245 \\
229 \\
205 \\
177 \\
152 \\
127 \\
104 \\
82\end{array}$ & $\begin{array}{r}\$ 95 \\
132 \\
173 \\
234 \\
300 \\
328 \\
347 \\
348 \\
331 \\
305 \\
265 \\
227 \\
196 \\
162 \\
127 \\
97\end{array}$ & $\begin{array}{r}\$ 88 \\
122 \\
161 \\
227 \\
300 \\
334 \\
352 \\
354 \\
340 \\
309 \\
272 \\
233 \\
191 \\
158 \\
120 \\
91\end{array}$ & $\begin{array}{r}\$ 92 \\
128 \\
168 \\
231 \\
300 \\
331 \\
348 \\
350 \\
332 \\
304 \\
265 \\
226 \\
192 \\
159 \\
124 \\
94\end{array}$ \\
\hline Number of estimates.. & 55 & 44 & 114 & 98 & 43 & 148 \\
\hline
\end{tabular}

1 North Central States: Ohio, Indiana, Mlinois, Michigan, Wisconsin, Minnesota, North Dakota, South Dakota, Iowa, Nebraska, Kansas, and Missouri.

2 Northeastern States: New England States, New York, New Jersey, and Pennsylvania.

\section{GUERNSEYS.}

Table II shows the influence of age on the values of four classes of Guernsey cows, worth per head $\$ 80, \$ 100, \$ 200$, and $\$ 300$, respectively, at three years of age. The estimates are classified as in Table I. These figures, as compared with those in Table I, indicate that appreciation and depreciation due to age are about the same for Guernseys and Holsteins. According to these estimates, Guernsey calves and old cows, like those of the Holsteins, are cheaper in the East than in the Central West. In the case of pure breds in their prime, however, the reverse is true. The estimates indicate that Guernsey cows of all classes reach their highest value at 5 and 6 years of age. (See fig. 2.) 


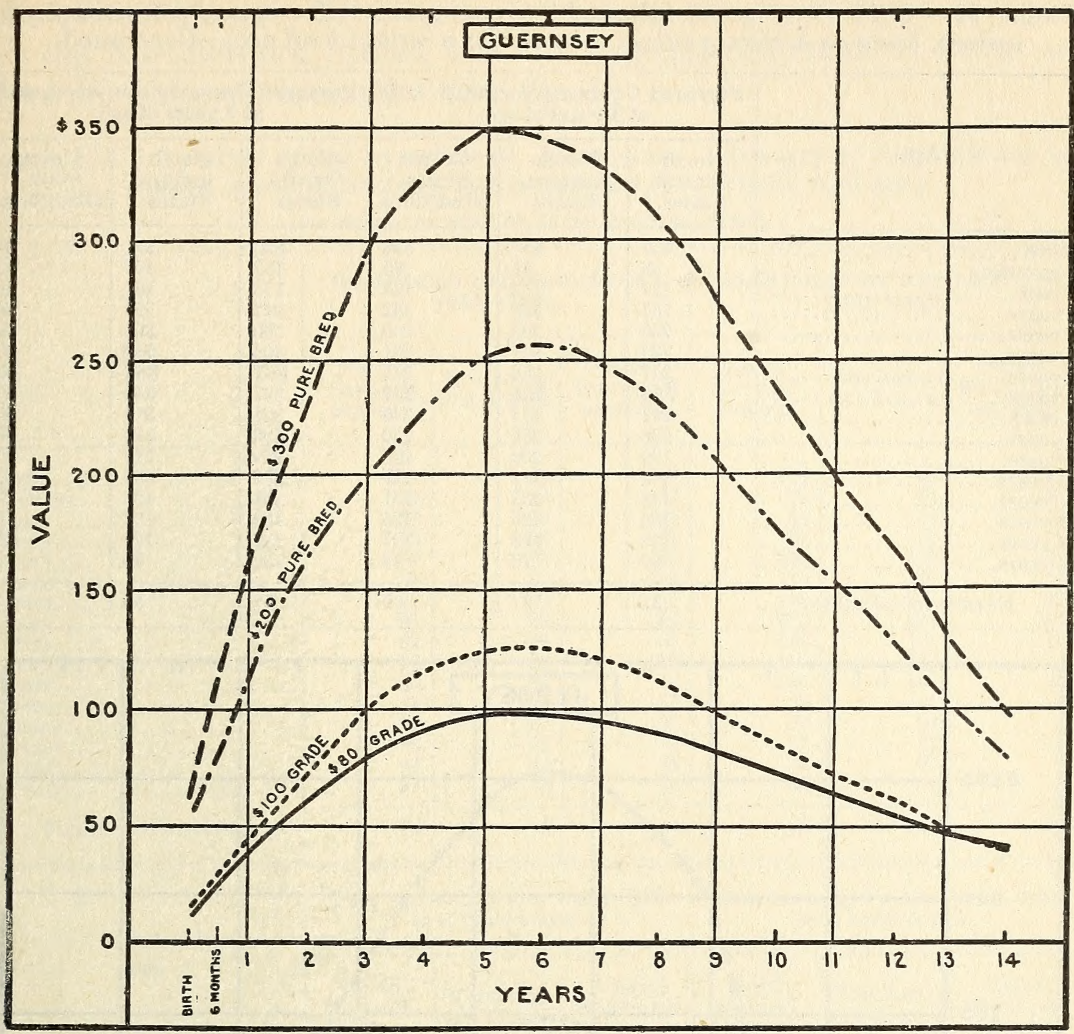

FIG. 2.-Curves showing influence of age on values of Guernsey cows. (Averages of 389 estimates by owners, based on arbitrary values assumed for a certain fixed age.)

TABLE II.-Influence of age on values of Guernsey cows. (Averages of 389 estimates by owners, based on arbitrary values assumed for a certain fixed age.)

[Averages as reported to the nearest dollar.]

\begin{tabular}{|c|c|c|c|c|c|c|}
\hline \multirow{2}{*}{ Age. } & \multicolumn{3}{|c|}{$\begin{array}{c}\text { Grade Guernsey cow worth } \$ 80 \text { at } \\
3 \text { years of age. }\end{array}$} & \multicolumn{3}{|c|}{$\begin{array}{c}\text { Grade Guernsey cow worth } \$ 100 \text { at } \\
3 \text { years of age. }\end{array}$} \\
\hline & $\begin{array}{l}\text { North } \\
\text { Central } \\
\text { States. }\end{array}$ & $\begin{array}{l}\text { North- } \\
\text { eastern } \\
\text { States. }\end{array}$ & $\begin{array}{c}\text { United } \\
\text { States, } \\
\text { all sections. }\end{array}$ & $\begin{array}{l}\text { North } \\
\text { Central } \\
\text { States. }\end{array}$ & $\begin{array}{l}\text { North- } \\
\text { eastern } \\
\text { States. }\end{array}$ & $\begin{array}{c}\text { United } \\
\text { States, } \\
\text { all sections. }\end{array}$ \\
\hline 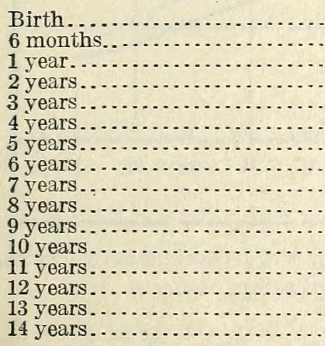 & $\begin{array}{r}\$ 11 \\
25 \\
40 \\
61 \\
80 \\
92 \\
99 \\
100 \\
97 \\
91 \\
83 \\
73 \\
65 \\
57 \\
50 \\
44\end{array}$ & $\begin{array}{r}\$ 10 \\
21 \\
38 \\
58 \\
80 \\
90 \\
\mathbf{9 8} \\
\mathbf{9 8} \\
96 \\
91 \\
81 \\
72 \\
63 \\
53 \\
46 \\
39\end{array}$ & $\begin{array}{r}\$ 11 \\
24 \\
40 \\
61 \\
80 \\
91 \\
\mathbf{9 9} \\
\mathbf{9 9} \\
96 \\
90 \\
82 \\
73 \\
64 \\
55 \\
48 \\
42\end{array}$ & $\begin{array}{r}\$ 15 \\
30 \\
48 \\
74 \\
100 \\
116 \\
127 \\
128 \\
123 \\
115 \\
100 \\
87 \\
74 \\
63 \\
52 \\
42\end{array}$ & $\begin{array}{r}\$ 11 \\
25 \\
42 \\
70 \\
100 \\
118 \\
126 \\
128 \\
121 \\
112 \\
97 \\
84 \\
69 \\
59 \\
47 \\
40\end{array}$ & $\begin{array}{r}\$ 13 \\
27 \\
45 \\
72 \\
100 \\
117 \\
126 \\
127 \\
121 \\
111 \\
97 \\
85 \\
71 \\
61 \\
49 \\
41\end{array}$ \\
\hline Number of estimates.... & 57 & 39 & 101 & 45 & 52 & 103 \\
\hline
\end{tabular}


TABLE II.--Influence of age on values of Guernsey cows. (Averages of 389 estimates by owners, based on arbitrary values assumed for a certain fixed age)-Continued.

\begin{tabular}{|c|c|c|c|c|c|c|c|}
\hline & \multirow[b]{2}{*}{ Age. } & \multicolumn{3}{|c|}{$\begin{array}{l}\text { Purebred Guernsey cow worth } \$ 200 \\
\text { at } 3 \text { years of age. }\end{array}$} & \multicolumn{3}{|c|}{$\begin{array}{c}\text { Purebred Guernsey cow worth } \$ 300 \\
\text { at } 3 \text { years of age. }\end{array}$} \\
\hline & & $\begin{array}{l}\text { North } \\
\text { Central } \\
\text { States. }\end{array}$ & $\begin{array}{l}\text { North- } \\
\text { eastern } \\
\text { States. }\end{array}$ & $\begin{array}{c}\text { United } \\
\text { States, } \\
\text { all sections. }\end{array}$ & $\begin{array}{l}\text { North } \\
\text { Central } \\
\text { States. }\end{array}$ & $\begin{array}{l}\text { North- } \\
\text { eastern } \\
\text { States. }\end{array}$ & $\begin{array}{c}\text { United } \\
\text { States, } \\
\text { all sections. }\end{array}$ \\
\hline \multicolumn{2}{|c|}{ 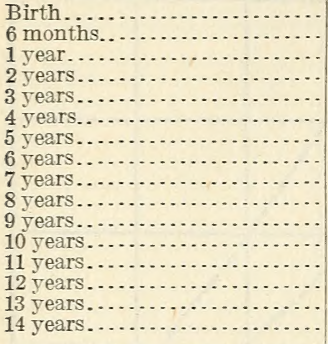 } & $\begin{array}{r}\$ 57 \\
88 \\
117 \\
164 \\
200 \\
229 \\
247 \\
250 \\
241 \\
226 \\
199 \\
175 \\
148 \\
126 \\
106 \\
87\end{array}$ & $\begin{array}{r}\$ 55 \\
81 \\
112 \\
160 \\
200 \\
232 \\
258 \\
264 \\
254 \\
235 \\
209 \\
182 \\
155 \\
125 \\
98 \\
70\end{array}$ & $\begin{array}{r}\$ 56 \\
85 \\
115 \\
162 \\
200 \\
231 \\
251 \\
257 \\
246 \\
230 \\
203 \\
179 \\
151 \\
126 \\
101 \\
78\end{array}$ & $\begin{array}{l}\$ 90 \\
130 \\
173 \\
242 \\
300 \\
324 \\
337 \\
333 \\
321 \\
299 \\
269 \\
232 \\
201 \\
169 \\
137 \\
107\end{array}$ & $\begin{array}{r}\$ 71 \\
107 \\
157 \\
224 \\
300 \\
340 \\
363 \\
360 \\
343 \\
316 \\
279 \\
239 \\
198 \\
162 \\
122 \\
90\end{array}$ & $\begin{array}{r}\$ 82 \\
120 \\
167 \\
234 \\
300 \\
332 \\
\mathbf{3 5 0} \\
346 \\
332 \\
307 \\
273 \\
235 \\
199 \\
166 \\
130 \\
99\end{array}$ \\
\hline \multicolumn{2}{|c|}{ Number of estimates.... } & 31 & 34 & 70 & 57 & 50 & 115 \\
\hline
\end{tabular}

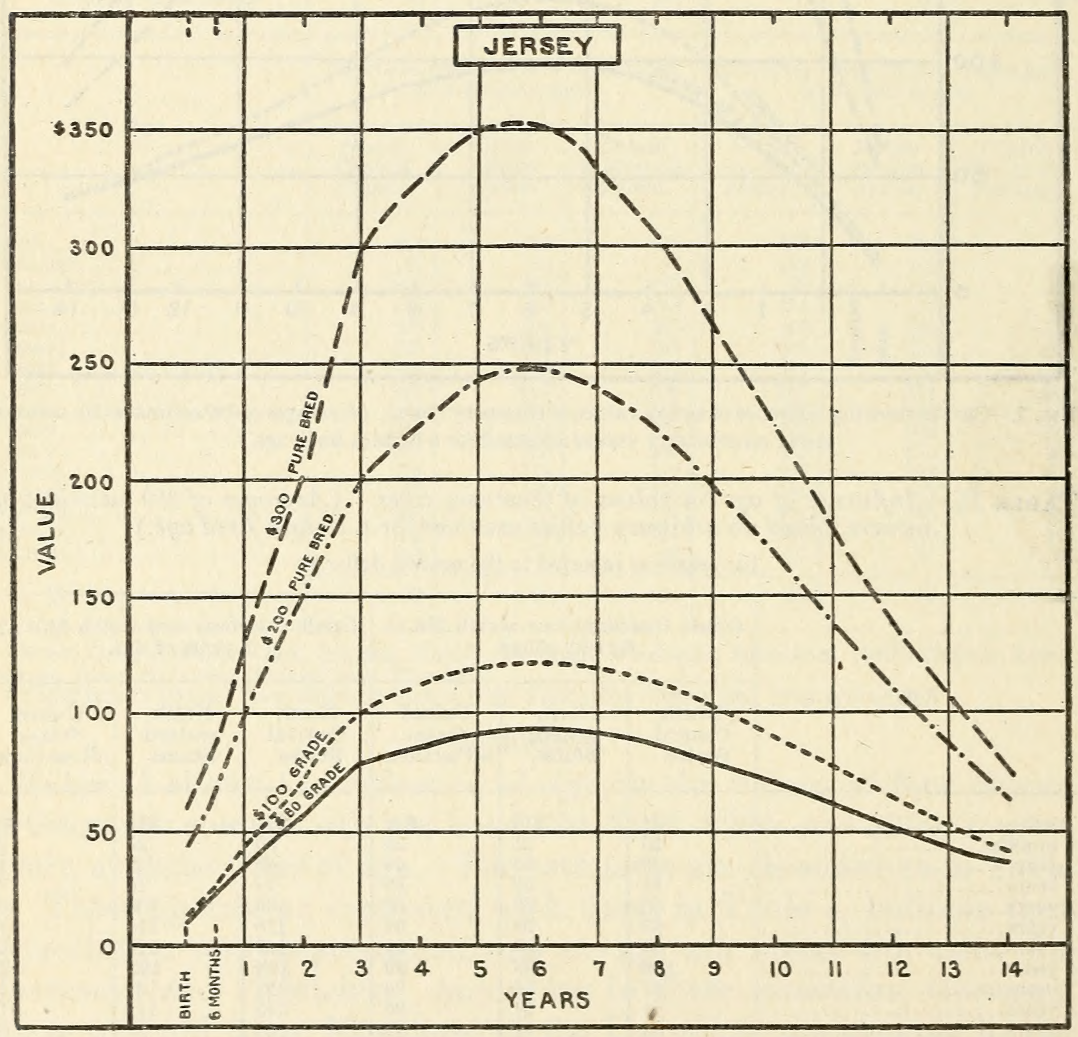

FIG. 3.-Curves showing influence of age on values of Jersey cows. (Averages of 511 estimates by owners, based on arbitrary values assumed for a certain fixed age.)

\section{JERSEYS.}

In Table III the estimates indicate that all classes of Jerseys are valued highest at 6 years, though the difference in value for 5 and 6 
years is not great. Here, again, calves are rated relatively cheaper for the Northeastern States, but the regional difference in value as given for old cows is small. (See fig. 3.)

TABLE III.-Influence of age on values of Jersey cows. (Averages of 511 estimates by owners, based on arbitrary values assumed for a certain fixed age.)

[Averages are reported to the nearest dollar.]

\begin{tabular}{|c|c|c|c|c|c|c|}
\hline \multirow{2}{*}{ Age. } & \multicolumn{3}{|c|}{$\begin{array}{c}\text { Grade Jersey cow worth } \$ 80 \text { at } 3 \\
\text { years of age. }\end{array}$} & \multicolumn{3}{|c|}{$\begin{array}{c}\text { Grade Jersey cow worth } \$ 100 \text { at } 3 \\
\text { years of age. }\end{array}$} \\
\hline & $\begin{array}{l}\text { North } \\
\text { Central } \\
\text { States. }\end{array}$ & $\begin{array}{l}\text { Northeast- } \\
\text { ern States. }\end{array}$ & $\begin{array}{l}\text { United } \\
\text { States, all } \\
\text { sections. }\end{array}$ & $\begin{array}{l}\text { North } \\
\text { Central } \\
\text { States. }\end{array}$ & $\begin{array}{l}\text { Northeast- } \\
\text { ern States. }\end{array}$ & $\begin{array}{l}\text { United } \\
\text { States, all } \\
\text { sections. }\end{array}$ \\
\hline 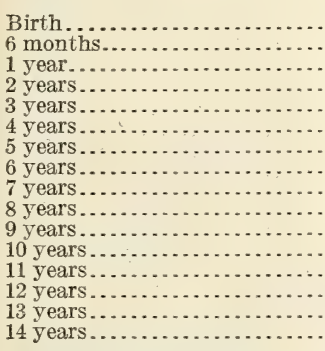 & $\begin{array}{r}\$ 11 \\
23 \\
37 \\
59 \\
80 \\
86 \\
91 \\
\mathbf{9 2} \\
92 \\
86 \\
80 \\
71 \\
62 \\
54 \\
44 \\
37\end{array}$ & $\begin{array}{l}\$ 8 \\
19 \\
32 \\
56 \\
80 \\
87 \\
93 \\
95 \\
93 \\
88 \\
82 \\
74 \\
62 \\
52 \\
42 \\
34\end{array}$ & $\begin{array}{r}\$ 10 \\
22 \\
35 \\
57 \\
80 \\
86 \\
91 \\
92 \\
91 \\
86 \\
79 \\
71 \\
61 \\
52 \\
43 \\
36\end{array}$ & $\begin{array}{r}\$ 13 \\
26 \\
44 \\
72 \\
100 \\
110 \\
119 \\
122 \\
121 \\
116 \\
105 \\
92 \\
79 \\
66 \\
54 \\
43\end{array}$ & $\begin{array}{r}\$ 9 \\
24 \\
42 \\
69 \\
100 \\
116 \\
124 \\
127 \\
126 \\
120 \\
109 \\
96 \\
84 \\
71 \\
57 \\
46\end{array}$ & $\begin{array}{r}\$ 12 \\
26 \\
44 \\
72 \\
100 \\
111 \\
119 \\
121 \\
119 \\
113 \\
102 \\
89 \\
77 \\
64 \\
52 \\
41\end{array}$ \\
\hline Number of estimates.... & 72 & 24 & 144 & 69 & 25 & 134 \\
\hline
\end{tabular}

\begin{tabular}{|c|c|c|c|c|c|c|}
\hline \multirow[b]{2}{*}{ Age. } & \multicolumn{3}{|c|}{$\begin{array}{c}\text { Purebred Jersey cow worth } \$ 200 \\
\text { at } 3 \text { years of age. }\end{array}$} & \multicolumn{3}{|c|}{$\begin{array}{c}\text { Purebred Jersey cow worth } \$ 300 \\
\text { at } 3 \text { years of age. }\end{array}$} \\
\hline & $\begin{array}{l}\text { North } \\
\text { Central } \\
\text { States. }\end{array}$ & $\begin{array}{l}\text { Northeast- } \\
\text { ern States. }\end{array}$ & $\begin{array}{l}\text { United } \\
\text { States, all } \\
\text { sections. }\end{array}$ & $\begin{array}{l}\text { North } \\
\text { Central } \\
\text { States. }\end{array}$ & $\begin{array}{l}\text { Northeast- } \\
\text { ern States. }\end{array}$ & $\begin{array}{l}\text { United } \\
\text { States, all } \\
\text { sections. }\end{array}$ \\
\hline 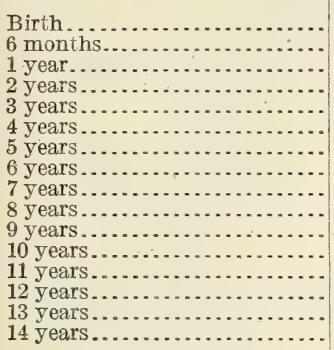 & $\begin{array}{r}\$ 42 \\
68 \\
103 \\
149 \\
200 \\
228 \\
247 \\
254 \\
245 \\
228 \\
204 \\
179 \\
144 \\
118 \\
89 \\
70\end{array}$ & $\begin{array}{r}\$ 41 \\
61 \\
94 \\
147 \\
200 \\
231 \\
256 \\
269 \\
261 \\
247 \\
217 \\
187 \\
151 \\
124 \\
94 \\
67\end{array}$ & $\begin{array}{r}\$ 44 \\
68 \\
103 \\
152 \\
200 \\
226 \\
245 \\
249 \\
240 \\
223 \\
196 \\
169 \\
138 \\
112 \\
87 \\
64\end{array}$ & $\begin{array}{r}\$ 54 \\
86 \\
129 \\
207 \\
300 \\
337 \\
359 \\
\mathbf{3 6 4} \\
346 \\
317 \\
275 \\
229 \\
190 \\
151 \\
106 \\
71\end{array}$ & $\begin{array}{r}\$ 50 \\
80 \\
124 \\
205 \\
300 \\
322 \\
353 \\
\mathbf{3 5 5} \\
336 \\
312 \\
267 \\
225 \\
\mathbf{1 8 3} \\
152 \\
112 \\
81\end{array}$ & $\begin{array}{r}\$ 59 \\
89 \\
133 \\
207 \\
300 \\
330 \\
350 \\
351 \\
332 \\
305 \\
266 \\
222 \\
178 \\
140 \\
105 \\
74\end{array}$ \\
\hline Number of estimates.... & 52 & 34 & 137 & 31 & $\overline{16}$ & $\overline{96}$ \\
\hline
\end{tabular}

AYRSHIRES.

According to the estimates averaged in Table IV, Ayrshire calves and old cows are cheaper in the Northeastern States than in the North Central States, while Ayrshire cows during their period of highest production are a little higher in the East. The values placed on old cows are exceptionally high for the North Central States. According to the estimates all classes of Ayrshires reach their maximum value at 6 years. (See fig. 4.) 


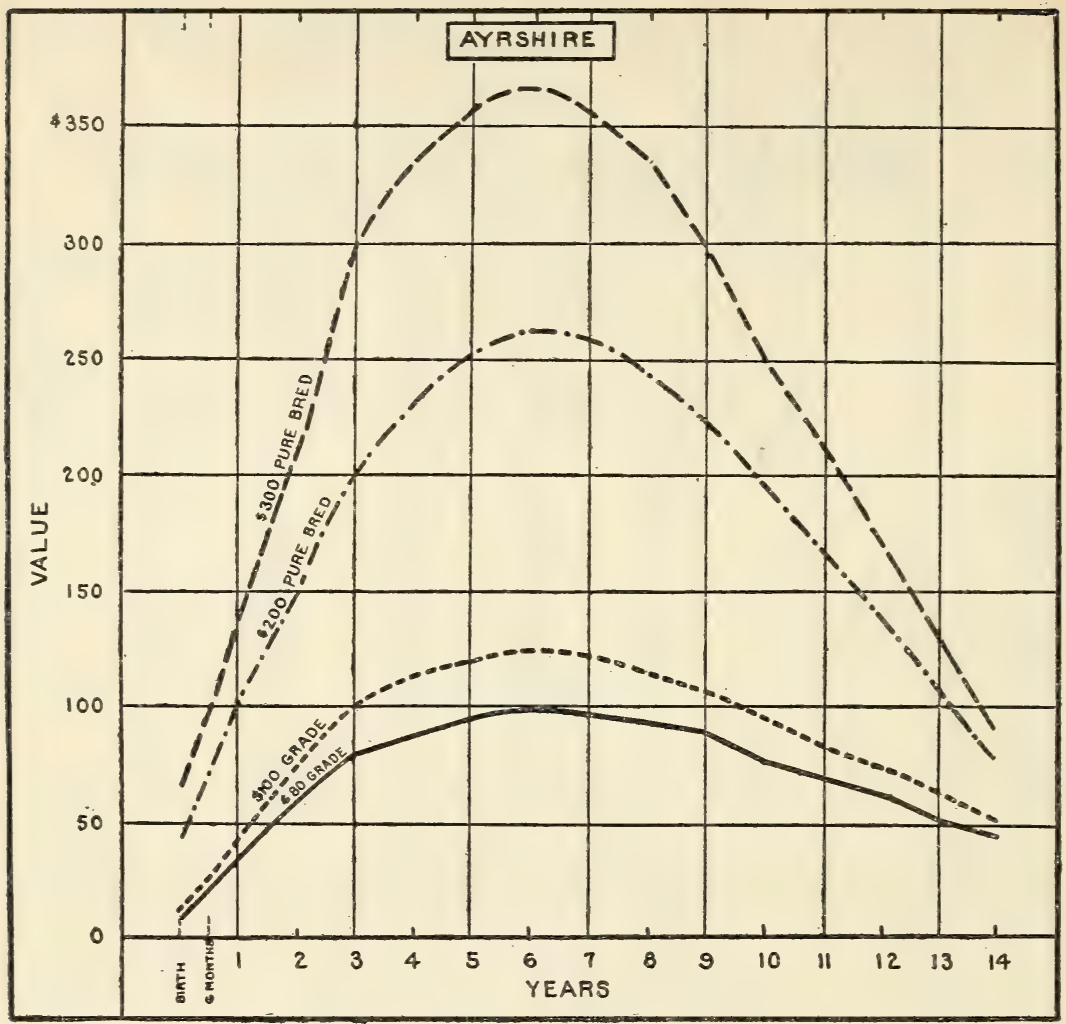

FIG. 4.-Curves showing influence of age on values of Ayrshire cows (Averages of 416 estimates by owners, based on arbitrary values assumed for a certain fixed age.)

TABLE IV.- Influence of age on values of Ayrshire cows. (Averages of 416 estimates by owners, based on arbitrary values assumed for a certain fixed age.)

(Averages are reported to the nearest dollar.)

Grade Ayrshire cow worth $\$ 80$ at 3 Grade Ayrshire cow worth $\$ 100$ at 3 years of age.

Age.

\begin{tabular}{l|l|c|c|c|c}
\hline North & North- & United & North & North- & United \\
Central & eastern & States, all & Central & eastern & States, al \\
States. & States. & sections. & States. & States. & Sections.
\end{tabular}

\begin{tabular}{|c|c|c|c|c|c|c|}
\hline 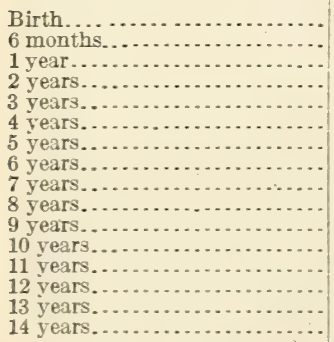 & $\begin{array}{r}\$ 11 \\
23 \\
37 \\
59 \\
80 \\
87 \\
91 \\
95 \\
94 \\
90 \\
85 \\
78 \\
69 \\
62 \\
54 \\
47\end{array}$ & $\begin{array}{r}\$ 8 \\
20 \\
33 \\
55 \\
80 \\
89 \\
96 \\
100 \\
100 \\
96 \\
88 \\
79 \\
71 \\
61 \\
51 \\
43\end{array}$ & $\begin{array}{l}\$ 9 \\
20 \\
36 \\
56 \\
80 \\
87 \\
95 \\
\mathbf{9 9} \\
98 \\
94 \\
89 \\
78 \\
70 \\
61 \\
52 \\
45\end{array}$ & $\begin{array}{r}\$ 15 \\
30 \\
48 \\
76 \\
100 \\
109 \\
114 \\
116 \\
114 \\
108 \\
99 \\
90 \\
79 \\
69 \\
60 \\
52\end{array}$ & $\begin{array}{r}\$ 11 \\
26 \\
42 \\
72 \\
100 \\
111 \\
120 \\
125 \\
123 \\
118 \\
108 \\
96 \\
84 \\
73 \\
61 \\
50\end{array}$ & $\begin{array}{r}\$ 12 \\
26 \\
43 \\
73 \\
100 \\
111 \\
119 \\
123 \\
121 \\
116 \\
106 \\
95 \\
83 \\
73 \\
61 \\
51\end{array}$ \\
\hline Number of estimates.... & 25 & 83 & 137 & 26 & 107 & 141 \\
\hline
\end{tabular}


TABLE IV.-Influence of age on values of Ayrshire cows. (Averages of 416 estimates by owners, based on arbitråry values assumed for a certain fixed age)-Continued.

\begin{tabular}{|c|c|c|c|c|c|c|}
\hline \multirow{2}{*}{ Age. } & \multicolumn{3}{|c|}{$\begin{array}{l}\text { Purebred Ayrshire cow worth } \$ 200 \\
\text { at } 3 \text { years of age. }\end{array}$} & \multicolumn{3}{|c|}{$\begin{array}{c}\text { Purebred Ayrshire cow worth } \$ 300 \\
\text { at } 3 \text { years of age. }\end{array}$} \\
\hline & $\begin{array}{l}\text { North } \\
\text { Central } \\
\text { States. }\end{array}$ & $\begin{array}{l}\text { North- } \\
\text { eastern } \\
\text { States. }\end{array}$ & $\begin{array}{l}\text { United } \\
\text { States, all } \\
\text { sections. }\end{array}$ & $\begin{array}{l}\text { North } \\
\text { Central } \\
\text { States. }\end{array}$ & $\begin{array}{l}\text { North- } \\
\text { eastern } \\
\text { States. }\end{array}$ & $\begin{array}{l}\text { United } \\
\text { States, all } \\
\text { sections. }\end{array}$ \\
\hline 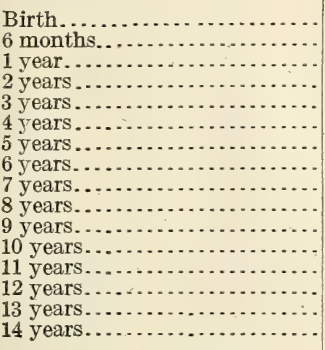 & $\begin{array}{r}\$ 44 \\
73 \\
108 \\
155 \\
200 \\
233 \\
243 \\
250 \\
245 \\
233 \\
209 \\
191 \\
166 \\
142 \\
113 \\
87\end{array}$ & $\begin{array}{r}\$ 42 \\
68 \\
101 \\
148 \\
200 \\
228 \\
255 \\
\mathbf{2 6 6} \\
265 \\
252 \\
229 \\
202 \\
168 \\
132 \\
99 \\
74\end{array}$ & $\begin{array}{r}\$ 44 \\
70 \\
103 \\
150 \\
200 \\
230 \\
253 \\
\mathbf{2 6 2} \\
258 \\
244 \\
222 \\
196 \\
165 \\
134 \\
103 \\
78\end{array}$ & $\begin{array}{r}\$ 67 \\
99 \\
145 \\
224 \\
300 \\
322 \\
345 \\
\mathbf{3 5 5} \\
342 \\
322 \\
290 \\
248 \\
210 \\
172 \\
130 \\
100\end{array}$ & $\begin{array}{r}\$ 65 \\
98 \\
138 \\
210 \\
300 \\
335 \\
361 \\
371 \\
360 \\
342 \\
299 \\
252 \\
212 \\
171 \\
130 \\
90\end{array}$ & $\begin{array}{r}\$ 66 \\
98 \\
140 \\
212 \\
300 \\
333 \\
357 \\
367 \\
355 \\
335 \\
256 \\
250 \\
210 \\
170 \\
129 \\
90\end{array}$ \\
\hline Number of estimates.... & 23 & 51 & 79 & 10 & 46 & 59 \\
\hline
\end{tabular}

GeNERAL Discussion OF PRECEDING TABLes.

With few exceptions, the preceding tables indicate that calves and old cows are cheaper in the Northeastern States, and that cows between the ages of 4 and 8 are cheaper in the North Central States. For all breeds, and for all classes of all breeds, the price of cows in health is greatest between 5 and 7 years, with the maximum usually at 6 . They are valued about the same at 4 as at 8 . The explanation for this may be that though the younger animals have before them a longer period of usefulness, the older ones have already demonstrated their worth. In all the tables the 14-year-old values for pure-bred cows are much greater than for grades. This may be partly due to higher average milk production, but it is undoubtedly more largely due to the possibility that these cows may yet produce valuable calves.

The cow worth $\$ 80$ at 3 years of age is given a birth value of about $\$ 10$, while the cow worth $\$ 100$ at that age is given a birth value of about $\$ 12$. Similarly the difference in value between the two classes of pure-bred cows at 3 years of age is $\$ 100$, while the difference in value at birth is in no case above $\$ 30$, leaving a margin of at least $\$ 70$ to cover interest on larger investment, risk of a greater possible loss, and any extra expense for feed or care. 
TABLE V.-Influence of age on values of a'ciiry cows, shown in per cent of maximum value. (Averages of 1,844 estimates by owners, based on arbitrary values assumed for a certain fixed age.)

\begin{tabular}{|c|c|c|c|c|c|c|c|c|}
\hline \multirow{2}{*}{ Age. } & \multicolumn{4}{|c|}{ Grade cows worth $\$ 80$ at 3 years of age. } & \multicolumn{4}{|c|}{ Grade cows worth $\$ 100$ at 3 years of age. } \\
\hline & Holstein. & Guernsey & Jersey. & Ayrshire. & Holstein. & Guernsey. & Jersey. & Ayrshire. \\
\hline 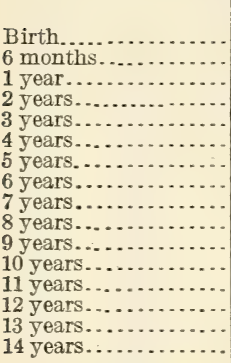 & $\begin{array}{r}\text { Per cent. } \\
10 \\
24 \\
38 \\
60 \\
78 \\
89 \\
98 \\
\mathbf{1 0 0} \\
97 \\
91 \\
82 \\
74 \\
66 \\
59 \\
51 \\
43\end{array}$ & \begin{tabular}{|r|} 
Per cent. \\
11 \\
24 \\
40 \\
62 \\
81 \\
92 \\
$\mathbf{1 0 0}$ \\
$\mathbf{1 0 0}$ \\
97 \\
91 \\
83 \\
$\mathbf{7 4}$ \\
65 \\
56 \\
48 \\
42
\end{tabular} & \begin{tabular}{|r} 
Per cent. \\
11 \\
24 \\
38 \\
62 \\
87 \\
94 \\
98 \\
$\mathbf{1 0 0}$ \\
98 \\
94 \\
82 \\
77 \\
66 \\
56 \\
47 \\
39
\end{tabular} & $\begin{array}{r}\text { Per cent. } \\
9 \\
20 \\
36 \\
57 \\
81 \\
88 \\
96 \\
\mathbf{1 0 0} \\
99 \\
95 \\
90 \\
79 \\
71 \\
62 \\
52 \\
45\end{array}$ & \begin{tabular}{|r} 
Per cent. \\
9 \\
21 \\
36 \\
59 \\
82 \\
92 \\
98 \\
100 \\
95 \\
88 \\
78 \\
68 \\
59 \\
51 \\
47 \\
39
\end{tabular} & $\begin{array}{r}\text { Per cent. } \\
10 \\
21 \\
35 \\
57 \\
79 \\
92 \\
99 \\
\mathbf{1 0 0} \\
95 \\
87 \\
76 \\
67 \\
56 \\
48 \\
39 \\
32\end{array}$ & \begin{tabular}{|r} 
Per cent. \\
10 \\
21 \\
36 \\
60 \\
83 \\
92 \\
98 \\
$\mathbf{1 0 0}$ \\
98 \\
93 \\
84 \\
74 \\
64 \\
53 \\
43 \\
34
\end{tabular} & $\begin{array}{r}\text { Per cent. } \\
10 \\
21 \\
35 \\
59 \\
81 \\
90 \\
97 \\
100 \\
98 \\
94 \\
86 \\
77 \\
67 \\
59 \\
50 \\
41\end{array}$ \\
\hline $\begin{array}{l}\text { Number of esti- } \\
\text { mates ........ }\end{array}$ & 107 & 101 & 144 & 137 & 159 & 103 & 134 & 141 \\
\hline
\end{tabular}

\begin{tabular}{|c|c|c|c|c|c|c|c|c|}
\hline \multirow{2}{*}{ Age. } & \multicolumn{4}{|c|}{$\begin{array}{c}\text { Purebred cows worth } \$ 200 \text { at } 3 \text { years } \\
\text { of age. }\end{array}$} & \multicolumn{4}{|c|}{$\begin{array}{c}\text { Purebred cows worth } \$ 300 \text { at } 3 \text { years } \\
\text { of age. }\end{array}$} \\
\hline & Holstein. & Guernsey. & Jersey. & Ayrshire. & Holstein. & Guernsey. & Jersey. & Ayrshire. \\
\hline 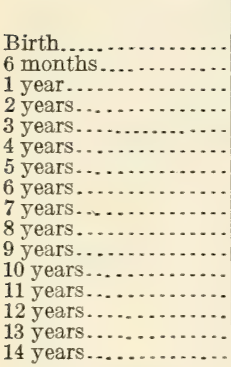 & $\begin{array}{r}\text { Per cent. } \\
24 \\
34 \\
46 \\
62 \\
78 \\
91 \\
98 \\
\mathbf{1 0 0} \\
96 \\
89 \\
80 \\
69 \\
59 \\
50 \\
41 \\
32\end{array}$ & \begin{tabular}{|r|} 
Per cent. \\
22 \\
33 \\
45 \\
63 \\
78 \\
90 \\
98 \\
$\mathbf{1 0 0}$ \\
96 \\
89 \\
79 \\
70 \\
59 \\
49 \\
39 \\
30
\end{tabular} & $\begin{array}{r}\text { Per cent. } \\
18 \\
27 \\
41 \\
61 \\
80 \\
91 \\
98 \\
100 \\
96 \\
90 \\
79 \\
68 \\
55 \\
45 \\
35 \\
26\end{array}$ & $\begin{array}{r}\text { Per cent. } \\
17 \\
27 \\
39 \\
57 \\
76 \\
88 \\
97 \\
\mathbf{1 0 0} \\
98 \\
93 \\
85 \\
75 \\
63 \\
51 \\
39 \\
30\end{array}$ & $\begin{array}{r}\text { Per cent. } \\
26 \\
36 \\
48 \\
66 \\
86 \\
94 \\
99 \\
100 \\
95 \\
87 \\
76 \\
64 \\
55 \\
45 \\
35 \\
27\end{array}$ & \begin{tabular}{|r|} 
Pér cent. \\
23 \\
34 \\
48 \\
67 \\
86 \\
95 \\
100 \\
99 \\
95 \\
88 \\
78 \\
67 \\
57 \\
47 \\
37 \\
28
\end{tabular} & $\begin{array}{r}\text { Per cent. } \\
17 \\
25 \\
38 \\
59 \\
85 \\
94 \\
99 \\
100 \\
94 \\
87 \\
76 \\
63 \\
51 \\
40 \\
30 \\
21\end{array}$ & $\begin{array}{r}\text { Per cent. } \\
18 \\
27 \\
38 \\
58 \\
82 \\
91 \\
97 \\
100 \\
97 \\
91 \\
81 \\
68 \\
57 \\
46 \\
35 \\
24\end{array}$ \\
\hline $\begin{array}{l}\text { Number of esti- } \\
\text { mates......... }\end{array}$ & 114 & 70 & 137 & 79 & 148 & 115 & 96 & 59 \\
\hline
\end{tabular}

Table $\mathrm{V}$ is a percentage table based on the data given in the preceding tables. It is believed that within reasonable limits the per cent columns will be found useful in estimating the values of other classes of dairy cows. For example, the per cent column for the cow worth $\$ 80$ at 3 years of age should be approximately accurate for the cow worth $\$ 70$ at 3 years of age, and the per cent column for the cow worth $\$ 100$ at 3 years of age should be approximately accurate for the cow worth $\$ 110$ at 3 years of age. On the other hand, the per cent column for the purebred cow worth $\$ 300$ at 3 years of age, could not very well be applied to cows valued at twice the amount at that age. 
All classes of live stock vary in actual value from time to time, but such variation does not greatly affect the relative value of the animal at different ages. A cow worth $\$ 100$ at 3 years of age when there is a great demand for dairy cattle will be worth less at the same age when there is smaller demand, but there will be a corresponding fluctuation in value for all other ages. It is, therefore, believed that the tables, especially the percentage table, will be found useful in determining relative value according to age.

Figures 1, 2, 3, and 4 present in graphic form the data for each class of all four breeds as given in Tables I, II, III, and IV. These curves indicate that the difference in value between the various classes of the same breed is greatest when the animals are in their prime and least for calves and old cows. It is also clearly shown that the curvature increases for each succeeding class from the $\$ 80$ grade to the $\$ 300$ purebred. This may be explained in part by saying that the price of grade cows is influenced largely by production, that the price of the $\$ 200$ purebred includes production and registration, and that in determining the price of the $\$ 300$ purebred three major factors must be considered, production, registration, and advanced registry.

\section{INFLUENCE OF AGE ON VALUE OF WORK HORSES.}

TABLE VI.-Influence of age on values of farm work horses. (Averages of 147 estimates by owners, based on an arbitrary value at prime.)

\begin{tabular}{|c|c|c|c|c|c|}
\hline Age. & $\begin{array}{l}\text { Maxi- } \\
\text { mum } \\
\text { value, } \\
\$ 250 \text {. }\end{array}$ & $\begin{array}{l}\text { Per cent } \\
\text { of maxi- } \\
\text { mum } \\
\text { value. }\end{array}$ & Age. & $\begin{array}{l}\text { Maxi- } \\
\text { mum } \\
\text { value, } \\
\$ 250 \text {. }\end{array}$ & $\begin{array}{l}\text { Per cent } \\
\text { of maxi- } \\
\text { mum } \\
\text { value. }\end{array}$ \\
\hline 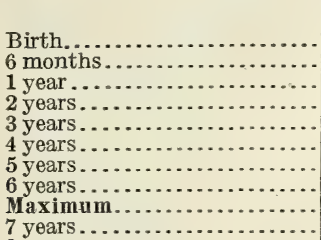 & $\begin{array}{r}\$ 39 \\
63 \\
96 \\
142 \\
185 \\
220 \\
242 \\
248 \\
\mathbf{2 5 0} \\
247\end{array}$ & $\begin{array}{r}\text { Per cent. } \\
16 \\
25 \\
38 \\
57 \\
74 \\
88 \\
97 \\
99 \\
100 \\
99\end{array}$ & 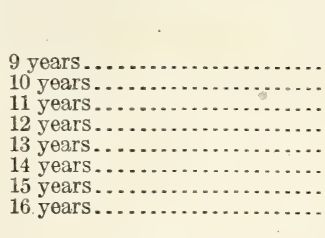 & $\begin{array}{l}227 \\
209 \\
190 \\
174 \\
154 \\
137 \\
119 \\
101\end{array}$ & $\begin{array}{r}\text { Per cent. } \\
91 \\
84 \\
76 \\
70 \\
62 \\
55 \\
48 \\
40\end{array}$ \\
\hline years & 24 & 96 & Number of estimates... & 147 & \\
\hline
\end{tabular}

Table VI shows the estimated influence of age on the value of farm work horses, and the per cent that the value at any age is of the maximum value. The estimates are for the farm horse worth $\$ 250$ in his prime. This is a value much above that of the average farm work horse, but the per cent columns may be used within reasonable limits in determining the influence of age on the values of cheaper farm horses. According to these figures the farm work horse reaches his maximum value at some point between six and seven years of age. 
If still in health his value at 16 years is 40 per cent of his maximum value. At birth he is worth 16 per cent of his maximum value, and there is little change in value between the ages of 5 and 9.

Figure 5 presents in graphic form the data given in Table VI. This curve differs in some respects from the curves for purebred

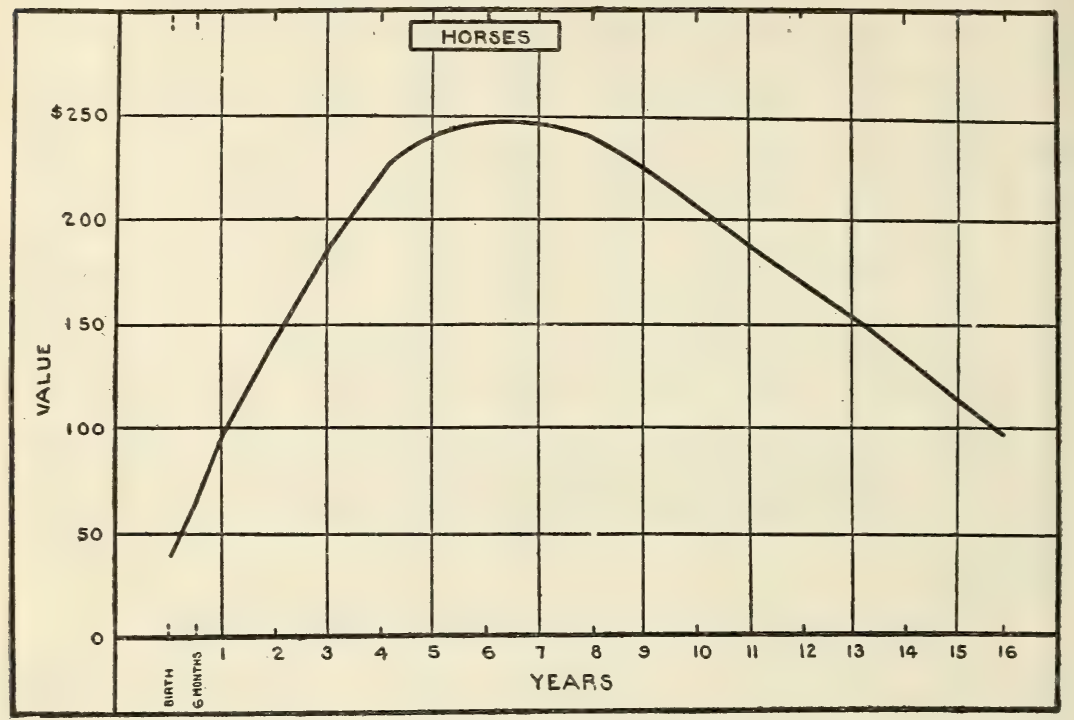

FiG. 5.-Curve showing the influence of age on the values of farm work horses. (Averages of 147 estimates by owners.)

dairy cows and is altogether different from the curves for grade dairy cows. As compared with the values given for pure-bred dairy cows of about the same maximum value, the birth value of horses is considerably less, the age of maturity a little later, and the old-age value somewhat higher. 





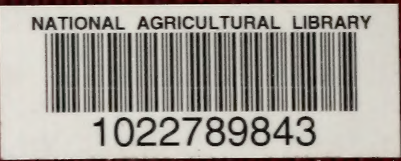

